\title{
Obesity Paradox in Chronic Liver Diseases: Product of Bias or a Real Thing?
}

\author{
Ines Bilic Curcic ${ }^{1,2}$, Maja Cigrovski Berkovic ${ }^{3}$, Lucija Kuna ${ }^{4}$, Hrvoje Roguljic ${ }^{1,5}$, \\ Robert Smolic $^{6,7}$, Silvija Canecki Varzic ${ }^{2,8}$, Lucija Virovic Jukic ${ }^{9}$ and Martina Smolic*1,4 \\ ${ }^{1}$ Department of Pharmacology, Faculty of Medicine, J. J. Strossmayer University of Osijek, Osijek, Croatia; ${ }^{2}$ Department of \\ Diabetes, Endocrinology and Metabolism Disorders, Osijek University Hospital Centre, Faculty of Medicine, Josip Juraj \\ Strossmayer University of Osijek, Osijek, Croatia; ${ }^{3}$ Department for Endocrinology, Diabetes and Metabolism University Hospital \\ Center, "Sestre Milosrdnice", Zagreb, Croatia; ${ }^{4}$ Department of Pharmacology and Biochemistry, Faculty of Dental Medicine and \\ Health, J. J. Strossmayer University of Osijek, Osijek, Croatia; ${ }^{5}$ Department for Cardiovascular Disease, Osijek University \\ Hospital, Osijek, Croatia; ${ }^{6}$ Department of Gastroenterology and Hepatology, Osijek University Hospital Centre, Faculty of \\ Medicine, Josip Juraj Strossmayer University of Osijek, Osijek, Croatia; ${ }^{7}$ Department of Pathophysiology, Faculty of Medicine, \\ Josip Juraj Strossmayer University of Osijek, Osijek, Croatia; ${ }^{8}$ Department of Internal Medicine, Faculty of Medicine, Josip Juraj \\ Strossmayer University of Osijek, Osijek, Croatia; ${ }^{9}$ Department of Gastroenterology and Hepatology, Sisters of Mercy University
} Hospital, University of Zagreb School of Medicine, Zagreb, Croatia

\begin{abstract}
In recent years, evidence supporting the theory of obesity paradox has increased, showing that obese/overweight people with prevalent chronic diseases experience lower mortality compared with patients of normal weight. So far, evidence is most comprehensive in cardiovascular and chronic renal diseases; however, published studies are prone to many biases, enabling us to reach a definite conclusion. Available data in chronic liver disease is scarce and ambiguous. Obesity is traditionally associated with nonalcoholic fatty liver disease and steatosis in viral hepatitis and as such one would not expect the obesity paradox to be a real possibility in liver disease. Yet, there seem to be new data indicating the opposite - the obesity paradox exists in severe and end-stage liver cirrhosis, which could be attributed to a better lean mass in patients with higher body mass index, meaning that sarcopenia, as one of the most important prognostic factors of survival, is less likely to be present. Nonetheless, the problem of various methodological problems addressing the association between body weight and mortality, which is present both in liver disease and other chronic diseases, are preventing us from attaining an unanimous conclusion. Still, we should be aware that the obesity paradox might be true, especially in severe and end-stage illness. This suggests focusing our efforts toward preserving or building up fat-free mass and decreasing inflammatory activity responsible for catabolism and sarcopenia, and implying that the underlaying cause should be treated.
\end{abstract}

Citation of this article: Curcic IB, Berkovic MC, Kuna L, Roguljic H, Smolic R, Varzic SC, et al. Obesity paradox in chronic

Keywords: Obesity paradox; Chronic liver disease; NAFLD; NASH; Cirrhosis. Abbreviations: BMI, body mass index; HCC, hepatocellular carcinoma; NAFLD, nonalcoholic fatty liver disease; NASH, nonalcoholic steatohepatitis; NHANES, National Health and Nutrition Examination Survey.

Received: 11 July 2019; Revised: 8 September 2019; Accepted: 9 September 2019

* Correspondence to: Martina Smolic, Department of Pharmacology, J. J. Strossmayer University of Osijek Faculty of Medicine Osijek, J. Huttlera 4, Osijek 31000, Croatia. Tel: + 385-31-512-800, Fax: +385-31-512-833, E-mail: martina.smolic@ mefos.hr liver diseases: Product of bias or a real thing? J Clin Transl Hepatol 2019;7(3):275-279. doi: 10.14218/JCTH.2019.00029.

\section{Introduction}

Obesity, among other diseases and conditions, increases morbidity from cardiovascular disease, diabetes, some cancer types, biliary disease, and arthrosis, and impairs quality of life, representing one of the most important causes of mortality. ${ }^{1}$ Global prevalence of obesity (defined by body mass index (BMI) of $\geq 30 \mathrm{~kg} / \mathrm{m}^{2}$ ) and overweight (defined by BMI of 25.0 to $29.9 \mathrm{~kg} / \mathrm{m}^{2}$ ) has reached epidemic proportions. According to 2016 World Health Organization data, $39 \%$ of adults were overweight and $13 \%$ (over 650 million) were obese; the numbers, although already disturbing, might be an underestimation of the true prevalence. ${ }^{2}$

On the other hand, evidence is increasingly showing that obese/overweight people with prevalent chronic diseases experience lower mortality compared with patients of normal weight during acute illnesses. For instance, in one metaanalysis, in patients with chronic heart failure, obesity was associated with a $33 \%$ lower risk of mortality relative to those with normal weight, while in patients with ischemic heart disease, similar risk reductions have also been observed. ${ }^{3-6}$

This paradoxical observation, known as the "obesity paradox," is rather confusing since lifestyle intervention with a goal of achieving weight reduction is a fundamental issue in the management of these conditions. ${ }^{7-9}$ Possible consequences of the obesity paradox in chronic diseases could have quite an impact on public health and clinical practice. If the obesity paradox proves to be true, the recommendation of change of lifestyle with the goal of weight loss in the current guidelines regarding those conditions, could actually do more harm than good. ${ }^{10}$ Also, in the national guidelines regarding weight loss in the overall population, no distinction has been made between various conditions in which obesity could be protective.

So, it is important to elucidate the true obesity-related survival benefit, especially in the era of the current wisdom of weight loss. ${ }^{11}$ One of the crucial problems we are facing today 
is understanding the definition of obesity, or rather addressing lack of the same. It is of utter importance to revisit the definition of obesity as made by the sole use of BMI as a measure, since it lacks information on body composition, such as sarcopenia, which might be clinically relevant in a condition within the obesity paradox. ${ }^{12-14}$ Furthermore, when discussing the obesity paradox, one must take into account different important confounders that lead to bias when interpreting data on the obesity-related health protective role in different disease settings.

The majority of available data related to the obesity paradox have been extracted from populations with overt diseases, wherein weight loss can be a sign of more severe disease or advanced disease stage. In a study examining myocardial infarction, congestive heart failure, stroke, and ischemic heart disease in a population-based study, the obesity paradox was observed to be prevalent in cardiovascular disease; however, incidence of cardiovascular disease provided no evidence of a survival advantage for obesity. ${ }^{6}$ In the case of the normal weight category including people who lost weight due to disease severity, then the adverse effects of obesity on mortality could be systematically and severely underestimated. ${ }^{15,16}$ Additionally, there is an issue of survivor bias, which relates to those who are obese and with more severe disease who die early, leaving behind more robust obese persons at the time of data analysis for obesity paradox investigation. So, in order to minimize and control the mentioned biases, additional studies examining persons at time of the incident disease, or using the prediagnosis weight or BMI data, would be more relevant prior to suggesting broad population guidelines on weight loss, without distinguishing between the persons with conditions/diseases where obesity might be protective. ${ }^{17}$

\section{Obesity paradox in nonalcoholic steatohepatitis - Possibility or improbability?}

One particular clinical entity requiring special attention when it comes to obesity and liver health is certainly nonalcoholic fatty liver disease (NAFLD), which has become an increasingly common cause of chronic liver disease in the developing world. In general, NAFLD has been found in $17-30 \%$ of the populations of Western nations and is estimated to be $2-4 \%$ worldwide. Metabolic disorders such as hypertension, type 2 diabetes mellitus, dyslipidemia and obesity show strongly associations with NAFLD. Moreover, NAFLD manifests as numerous series of liver disease, ranging from common steatosis to nonalcoholic steatohepatitis (NASH) and cirrhosis in a subset of patients. It is important to emphasize that not all patients with NASH will suffer from cirrhosis. Indeed, the collective NAFLD patients in who the disease will lead to NASH and require therapy is a hot area of investigation. Currently, various different therapeutic targets and numerous therapies are being investigated for NASH. ${ }^{18}$

Over the past few decades, obesity has emerged as a global epidemic, with significant influence on the increasing prevalence of obesity-related systemic disorders and involving NAFLD. ${ }^{19}$ This particularly refers to modifications in lifestyle, especially in physical activity and nutrition. In fact, it is well known that half of the adult population around the world, particularly in developed Western countries, have a problem with overweight or obesity. Although, obesity is one of the causes of a disease that can be prevented and which, if is uncontrolled, can lead to metabolic complications and consequently to premature mortality. ${ }^{20}$
Additionally, morbid obesity presents an increased risk for type 2 diabetes and cardiovascular disease, and is a significant risk factor for numerous malignant diseases, particularly hepatocellular carcinoma (HCC). ${ }^{21}$ It has been demonstrated that the increase in BMI $>30 \mathrm{~kg} / \mathrm{m}^{2}$ has influence on raising the risk of HCC development, particularly in the male population where HCC mortality rates are 5 times higher than in those with a normal BMI. ${ }^{22}$ Numerous essential studies related to control of inflammatory cytokine regeneration, adipose factors, oxidative stress, and lipid storage have explained NASH as a metabolic syndrome. Moreover, recent studies have also demonstrated the important role of genetic factors in contributing to NASH pathogenesis and to inducing malignancy in the liver. ${ }^{22}$ Furthermore, a connection has been made between obesity and NAFLD, such that obesity enhances the risk of progression of fibrosis and hepatic inflammation, leading to NASH-related cirrhosis. ${ }^{23}$

The pathophysiology of NASH is complex and still of major research interest. It is accepted that numerous mechanisms and pathways are included in the pathogenesis of NASH. Insulin resistance, dietary factors, genetic polymorphisms, and lipotoxicity form the group of mechanisms found to date and characterized as playing key roles in NASH pathogenesis. ${ }^{24}$ It remains uncertain, however, whether NASH always precedes NAFLD. Pathogenic driving factors are unlikely to be the same for all patients. Thus, the mechanisms that cause the disease and their clinical manifestations are greatly heterogeneous. ${ }^{25}$

A link between obesity, NAFLD and HCC is well established. Moreover, chronic infection with hepatitis B virus or hepatitis $C$ virus is strongly connected with hepatic steatosis. Compounded forms of metabolic syndrome involving type 2 diabetes mellitus, obesity and/or dyslipidemia are a frequent cause of viral hepatitis-associated hepatic steatosis. Due to that, damaged glucose metabolism enhances the possibility of hepatic failure and death in patients with recompensated hepatitis $C$ virus-related cirrhosis. ${ }^{26}$ In turn, hepatitis $C$ virus infection is essential as a risk factor, as it has significant influence on development of insulin resistance. 27,28 Therefore, based on these collective lines of evidence, the logical question to ask is whether it is possible that the obesity paradox even exists in the condition of liver disease.

\section{Obesity paradox in liver cirrhosis - Is it time for a paradigm shift?}

Answer to the question asked, could be provided by the data reported in a recently published study conducted by Kargozian et al. ${ }^{29}$ in which clinical outcomes, such as terms of mortality, length of stay, and total hospital charges, in hospitalized obese cirrhotic patients were compared with those of nonobese cirrhotic patients. The results were in alignment with the obesity paradox as indicated to be present in other intensive care patients. ${ }^{30,31}$ Obesity itself was associated with a decrease in inpatient mortality but with higher costs and lengthy hospitalization in patients with cirrhosis. However, the Kargozian study had several limitations, as it was of retrospective design and therefore susceptible to biases and with a lack of anthropometric data and BMI values for each patient. However, similar results were found in patients with hepatocellular carcinoma; specifically, lower BMI was a predictive factor for higher rates of mortality and shorter survival. Although, $20 \%$ of patients with lower BMI were actually undernourished, which could have produced alternate study 


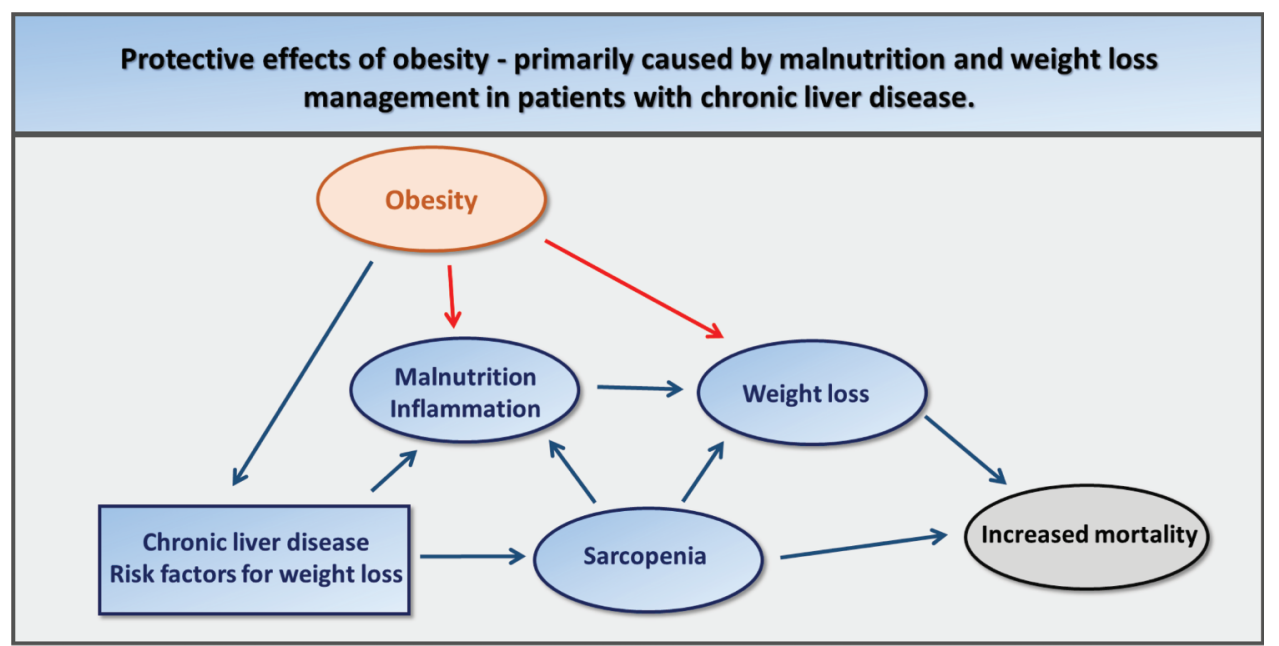

Fig. 1. Schematic presentation of obesity as a risk factor for chronic liver diseases, such as NASH and NAFLD. The latter are denoted by blue arrows (increasing impact). This risk factor could also have beneficial effects (denoted by red arrows; decreasing impact) on liver disease-associated mortality, which might be hampered when associated with sarcopenia, due to impact of sarcopenia on malnutrition and weight loss.

Abbreviations: NAFLD, nonalcoholic fatty liver disease; NASH, nonalcoholic steatohepatitis.

results considering that malnutrition in terminally ill patients is a poor prognostic risk factor. ${ }^{32}$

Previously published studies have suggested the complete opposite of the above data. In a study comprising data from the National Health and Nutrition Examination Survey (oft referred to as NHANES) database for patients without previous liver disease, cirrhosis-related deaths and hospitalizations were found to be positively associated with obesity (follow-up being 13 years). ${ }^{33}$ Recently, it was also found that increased BMI was positively associated with clinical decompensation in cirrhosis (with a mean follow-up of 59 months).$^{34}$ One of the possible explanations of those seemingly opposite results is regarding obesity as a consequence rather than a cause. This is supported by obese patients being more aggressively treated according to guidelines than normal body weight people, possibly contributing to a better outcome in obese patients. For example, obese patients are more likely to receive statins and oral antihyperglycemic agents, such as metformin, pioglitazone or liraglutide, contributing to a decrease in the inflammation processes and being associated with better prognosis in liver diseases. ${ }^{35-37}$ In one study including patients with chronic hepatitis $C$ virus-associated liver disease and severe liver fibrosis or compensated cirrhosis, $43 \%$ were obese and $32 \%$ were overweight. A $14 \%$ increase in the risk of worsening of liver fibrosis or decompensation was observed to be related to an increase in BMI (with a follow-up of 3.5 years). ${ }^{38}$

Support for the hypothesis of obesity paradox in liver disease was recently obtained by a study that demonstrated liver-related complications to be higher among patients with hepatitis $C$ cirrhosis than among patients with NAFLD cirrhosis, but overall mortality was the same. ${ }^{40}$ This finding was probably due to the higher BMI among NAFLD patients influencing a more prolonged course of illness compared to hepatitis $\mathrm{C}$ cirrhosis but with no difference in mortality. Also, NAFLD-related cirrhosis is factor of more slowly progressing disease than the other causes of cirrhosis (alcohol, chronic hepatitis C). In the initial stages, the nonobese patients may have other more aggressive cofactors for liver disease, thus affecting the final outcome. In addition, there is the factor of body composition; in older patients, BMI is a better indicator of lean body mass than the adipose tissue, whereas in patients with chronic illness obesity correlates with increased muscle mass and improved nutritional status. ${ }^{40,41}$

A well-known fact is that patients with advanced liver disease lose weight more prominently due to loss of appetite and inadequate nourishment caused by ascites and impaired gut motility; absorption of nutrients is also impaired due to bacterial overgrowth, while the inflammatory state induces anorexia, and glycogen stores decrease, thereby increasing gluconeogenesis. Those disturbances all lead to one common denominator: sarcopenia.42 A recent study on cirrhotic patients demonstrated that sarcopenic patients had increased mortality rates compared to nonsarcopenic patients. ${ }^{43}$ However, the prognosis was significantly worse in patients with sarcopenic obesity, followed by sarcopenia and visceral obesity; meanwhile, elderly (aged $\geq 64$ years) subjects had poorer prognosis that for sarcopenic obesity than did the subjects with normal body composition, suggesting once again that visceral obesity has a beneficial effect on prognosis as long there is no sarcopenia present. ${ }^{13}$

This leave us with the question of how the potential biases lurking around the corner could significantly affect the interpretation of the results from these collective studies? One of many possible answers is that among patients with already developed chronic liver disease, those with more serious conditions lose weight more prominently, thus creating a false image of the protective effect of obesity. Moreover, the answer could be that obese people with severe disease die earlier and a selectively overweight population of healthier people remain. ${ }^{6}$

\section{Conclusions}

Available data suggest that obesity prior to onset of liver disease could have detrimental effects, while higher BMI in the advanced liver disease may represent a positive prognostic factor; the latter is supported by evidence obtained from studies of other chronic illnesses, such as renal and 
cardiovascular disease, heart failure and rheumatoid arthritis. ${ }^{6,44-47}$ Thus, we can conclude that the fat mass itself is probably a factor associated with a better nutritional state positively affecting survival but not a relevant factor stipulating a better outcome. Although a protective role of obesity regarding survival or mortality rate cannot be established with certainty, the observation of patients with more severe and advanced liver disease without sarcopenia presenting higher BMI could mean a better prognosis (Fig. 1).

To summarize, there is an extensive body of evidence supporting the obesity paradox regarding other chronic illnesses, although some confounders may be present; however, data on liver diseases are still vague and scarce. Ambiguous results from the data published so far are preventing us from obtaining a unanimous conclusion. This difference is probably caused by several factors, including the widely used BMI as an imperfect tool for defining obesity, the use of prevalent models in published studies instead of incident ones since prevalent illness is inevitably influenced by weight loss caused by disease, and selective survival causing impartiality. However, we should be open to the possibility that the obesity paradox may be true and focus our efforts toward preserving or generating lean mass, such as that of muscle and bone, and decreasing inflammatory activity responsible for catabolism and sarcopenia; thus, the cause of the primary disease should be treated.

\section{Conflict of interest}

The authors have no conflict of interests related to this publication.

\section{Author contributions}

Conceived of and designed the article, and critically revised the manuscript (IBC, MS), performed critical revision of the manuscript for important intellectual content, obtained funding, and provided administrative, technical and material support (MS), performed literature searches and critical revision of the manuscript for important intellectual content (RS, SCV, LVJ), performed literature searches, wrote the manuscript, and updated the text of the paper (IBC, MCB, LK), performed literature searches and wrote the manuscript (HR).

\section{References}

[1] Lonardo A, Bellentani S, Argo CK, Ballestri S, Byrne CD, Caldwell SH, et al. Epidemiological modifiers of non-alcoholic fatty liver disease: Focus on highrisk groups. Dig Liver Dis 2015;47:997-1006. doi: 10.1016/j.dld.2015.08. 004 .

[2] de Herder WW, Eng C. Obesity, diabetes mellitus, and cancer. Endocr Relat Cancer 2012;19:E5-E7. doi: 10.1530/ERC-12-0264.

[3] Niedziela J, Hudzik B, Niedziela N, Gasior M, Gierlotka M, Wasilewski J, et al. The obesity paradox in acute coronary syndrome: a meta-analysis. Eur J Epidemiol 2014;29:801-812. doi: 10.1007/s10654-014-9961-9.

[4] Oreopoulos A, Padwal R, Norris CM, Mullen JC, Pretorius V, Kalantar-Zadeh K. Effect of obesity on short- and long-term mortality postcoronary revascularization: a meta-analysis. Obesity (Silver Spring) 2008;16:442-450. doi: 10.1038/oby.2007.36

[5] Romero-Corral A, Montori VM, Somers VK, Korinek J, Thomas RJ, Allison TG, et al. Association of bodyweight with total mortality and with cardiovascular events in coronary artery disease: a systematic review of cohort studies. Lancet 2006;368:666-678. doi: 10.1016/S0140-6736(06)69251-9.

[6] Chang VW, Langa KM, Weir D, Iwashyna TJ. The obesity paradox and incident cardiovascular disease: A population-based study. PLoS One 2017;12: e0188636. doi: 10.1371/journal.pone.0188636.
[7] Martín-Ponce E, Santolaria F, Alemán-Valls MR, González-Reimers E, MartínezRiera A, Rodríguez-Gaspar M, et al. Factors involved in the paradox of reverse epidemiology. Clin Nutr 2010;29:501-506. doi: 10.1016/j.clnu.2009.12.009.

[8] Han SJ, Boyko EJ. The evidence for an obesity paradox in type 2 diabetes mellitus. Diabetes Metab J 2018;42:179-187. doi: 10.4093/dmj.2018.0055.

[9] Curtis JP, Selter JG, Wang Y, Rathore SS, Jovin IS, Jadbabaie F, et al. The obesity paradox: body mass index and outcomes in patients with heart failure. Arch Intern Med 2005;165:55-61. doi: 10.1001/archinte.165.1.55.

[10] Smith SC Jr, Benjamin EJ, Bonow RO, Braun LT, Creager MA, Franklin BA, et al. AHA/ACCF secondary prevention and risk reduction therapy for patients with coronary and other atherosclerotic vascular disease: 2011 update: a guideline from the American Heart Association and American College of Cardiology Foundation endorsed by the World Heart Federation and the Preventive Cardiovascular Nurses Association. J Am Coll Cardiol 2011;58:2432-2446. doi: 10.1016/j.jacc.2011.10.824.

[11] Schmidt DS, Salahudeen AK. Obesity-survival paradox-still a controversy? Semin Dial 2007;20:486-492. doi: 10.1111/j.1525-139X.2007.00349.x.

[12] Pichard C, Kyle UG, Morabia A, Perrier A, Vermeulen B, Unger P. Nutritional assessment: lean body mass depletion at hospital admission is associated with an increased length of stay. Am J Clin Nutr 2004;79:613-618. doi: 10. 1093/ajcn/79.4.613.

[13] Roubenoff R. Sarcopenic obesity: the confluence of two epidemics. Obes Res 2004;12:887-888. doi: 10.1038/oby.2004.107.

[14] Lavie C], De Schutter A, Patel DA, Romero-Corral A, Artham SM, Milani RV. Body composition and survival in stable coronary heart disease: impact of lean mass index and body fat in the "obesity paradox". J Am Coll Cardiol 2012;60:1374-1380. doi: 10.1016/j.jacc.2012.05.037.

[15] Mehta NK, Chang V. The Oxford Handbook of the Social Science of Obesity. Cawley JH, editor. New York: Oxford University Press; 2011.

[16] Banack HR, Kaufman JS. Does selection bias explain the obesity paradox among individuals with cardiovascular disease? Ann Epidemiol 2015;25: 342-349. doi: 10.1016/j.annepidem.2015.02.008.

[17] World Health Organization. Obesity: preventing and managing the global epidemic. Report of a WHO. consultation (WHO Technical Report Series 894) 2000. Available from: https://www.who.int/nutrition/publications/obesity/ WHO_TRS_894/en/.

[18] Sarwar R, Pierce N, Koppe S. Obesity and nonalcoholic fatty liver disease: current perspectives. Diabetes Metab Syndr Obes 2018;11:533-542. doi 10.2147/DMSO.S146339.

[19] Renehan AG, Tyson M, Egger M, Heller RF, Zwahlen M. Body-mass index and incidence of cancer: a systematic review and meta-analysis of prospective observational studies. Lancet 2008;371:569-578. doi: 10.1016/S01406736(08)60269-X

[20] Larsson SC, Wolk A. Excess body fatness: an important cause of most cancers. Lancet 2008;371:536-537. doi: 10.1016/S0140-6736(08)60247-0.

[21] Wolk A, Gridley G, Svensson M, Nyrén O, McLaughlin JK, Fraumeni JF, et al. A prospective study of obesity and cancer risk (Sweden). Cancer Causes Control 2001;12:13-21. doi: 10.1023/A:1008995217664.

[22] Yu J, Shen J, Sun TT, Zhang X, Wong N. Obesity, insulin resistance, NASH and hepatocellular carcinoma. Semin Cancer Biol 2013;23:483-491. doi: 10. 1016/j.semcancer.2013.07.003.

[23] Jaycox SH. A fatty world: Exploring racial disparity in NAFLD/NASH. ] Hep 2016;2:2. doi: 10.21767/2471-9706.100014

[24] Chalasani N, Younossi Z, Lavine JE, Charlton M, Cusi K, Rinella M, et al. The diagnosis and management of nonalcoholic fatty liver disease: Practice guidance from the American Association for the Study of Liver Diseases. Hepatology 2018;67:328-357. doi: 10.1002/hep.29367.

[25] Alonso C, Fernández-Ramos D, Varela-Rey M, Martínez-Arranz I, Navasa N, Van Liempd SM, et al. Metabolomic identification of subtypes of nonalcoholic steatohepatitis. Gastroenterology 2017;152:1449-1461.e7. doi: 10.1053/j. gastro.2017.01.015.

[26] Calzadilla-Bertot L, Vilar-Gomez E, Torres-Gonzalez A, Socias-Lopez M, Diago M, Adams LA, et al. Impaired glucose metabolism increases risk of hepatic decompensation and death in patients with compensated hepatitis C virus-related cirrhosis. Dig Liver Dis 2016;48:283-290. doi: 10.1016/j. dld.2015.12.002

[27] Mehta SH, Brancati FL, Sulkowski MS, Strathdee SA, Szklo M, Thomas DL. Prevalence of type 2 diabetes mellitus among persons with hepatitis $C$ virus infection in the United States. Ann Intern Med 2000;133:592-599. doi: 10. 7326/0003-4819-133-8-200010170-00009.

[28] Sanyal AJ, Chalasani N, Kowdley KV, McCullough A, Diehl AM, Bass NM, et al. Pioglitazone, vitamin $\mathrm{E}$, or placebo for nonalcoholic steatohepatitis. N Engl J Med 2010;362:1675-1685. doi: 10.1056/NEJMoa0907929.

[29] Karagozian R, Bhardwaj G, Wakefield DB, Baffy G. Obesity paradox in advanced liver disease: obesity is associated with lower mortality in hospitalized patients with cirrhosis. Liver Int 2016;36:1450-1456. doi: 10. 1111/liv.13137.

[30] Hogue CW Jr, Stearns JD, Colantuoni E, Robinson KA, Stierer T, Mitter N, et al. The impact of obesity on outcomes after critical illness: a meta-analysis. Intensive Care Med 2009;35:1152-1170. doi: 10.1007/s00134-009-1424-5. 
[31] Akinnusi ME, Pineda LA, El Solh AA. Effect of obesity on intensive care morbidity and mortality: a meta-analysis. Crit Care Med 2008;36:151-158. doi: 10.1097/01.CCM.0000297885.60037.6E.

[32] Li Q, Xing H, Liu D, Li H. Negative impact of low body mass index on liver cirrhosis patients with hepatocellular carcinoma. World J Surg Oncol 2015; 13:294. doi: 10.1186/s12957-015-0713-4.

[33] Ioannou GN, Weiss NS, Kowdley KV, Dominitz JA. Is obesity a risk factor for cirrhosis-related death or hospitalization? A population-based cohort study. Gastroenterology 2003;125:1053-1059. doi: 10.1016/s0016-5085(03)01200-9.

[34] Berzigotti A, Garcia-Tsao G, Bosch J, Grace ND, Burroughs AK, Morillas R, et al. Obesity is an independent risk factor for clinical decompensation in patients with cirrhosis. Hepatology 2011;54:555-561. doi: 10.1002/hep. 24418.

[35] Souk K, Al-Badri M, Azar ST. The safety and benefit of statins in liver cirrhosis: a review. Exp Clin Endocrinol Diabetes 2015;123:577-580. doi: 10. 1055/s-0035-1564093.

[36] Donadon V, Balbi M, Mas MD, Casarin P, Zanette G. Metformin and reduced risk of hepatocellular carcinoma in diabetic patients with chronic liver disease. Liver Int 2010;30:750-758. doi: 10.1111/j.1478-3231.2010.02223.x.

[37] Blaslov K, Bulum T, Zibar K, Duvnjak L. Incretin based therapies: a nove treatment approach for non-alcoholic fatty liver disease. World J Gastroenterol 2014;20:7356-7365. doi: 10.3748/wjg.v20.i23.7356.

[38] Everhart JE, Lok AS, Kim HY, Morgan TR, Lindsay KL, Chung RT, et al. Weightrelated effects on disease progression in the hepatitis $C$ antiviral long-term treatment against cirrhosis trial. Gastroenterology 2009;137:549-557. doi: 10.1053/j.gastro.2009.05.007.

[39] Bhala N, Angulo P, van der Poorten D, Lee E, Hui JM, Saracco G, et al. The natural history of nonalcoholic fatty liver disease with advanced fibrosis or cirrhosis: an international collaborative study. Hepatology 2011;54:12081216. doi: 10.1002/hep.24491.
[40] Oreopoulos A, Ezekowitz JA, McAlister FA, Kalantar-Zadeh K, Fonarow GC, Norris CM, et al. Association between direct measures of body composition and prognostic factors in chronic heart failure. Mayo Clin Proc 2010;85:609617. doi: $10.4065 / \mathrm{mcp} .2010 .0103$.

[41] Casas-Vara A, Santolaria F, Fernández-Bereciartúa A, González-Reimers E, García-Ochoa A, Martínez-Riera A. The obesity paradox in elderly patients with heart failure: analysis of nutritional status. Nutrition 2012;28:616-622. doi: $10.1016 /$ j.nut.2011.10.006.

[42] Kim HY, Jang JW. Sarcopenia in the prognosis of cirrhosis: Going beyond the MELD score. World J Gastroenterol 2015;21:7637-7647. doi: 10.3748/wjg. V21.i25.7637.

[43] Hara N, Iwasa M, Sugimoto R, Mifuji-Moroka R, Yoshikawa K, Terasaka E, et al. Sarcopenia and sarcopenic obesity are prognostic factors for overall survival in patients with cirrhosis. Intern Med 2016;55:863-870. doi: 10. 2169/internalmedicine.55.5676.

[44] Lavie CJ, Milani RV Ventura HO Obesity and cardiovascular disease: risk factor, paradox, and impact of weight loss. J Am Coll Cardiol 2009;53: 1925-1932. doi: 10.1016/j.jacc.2008.12.068.

[45] Thomas F, Bean K, Pannier B, Oppert JM, Guize L, Benetos A. Cardiovascular mortality in overweight subjects: the key role of associated risk factors. Hypertension 2005;46:654-659. doi: 10.1161/01.HYP.0000184282.51550.00.

[46] De Schutter A, Lavie CJ, Patel DA, Milani RV. Obesity paradox and the heart: which indicator of obesity best describes this complex relationship? Curr Opin Clin Nutr Metab Care 2013;16:517-524. doi: 10.1097/MCO. Ob013e328363bcca.

[47] Elkan AC, Engvall IL, Cederholm T, Hafström I. Rheumatoid cachexia, central obesity and malnutrition in patients with low-active rheumatoid arthritis: feasibility of anthropometry, Mini Nutritional Assessment and body composition techniques. Eur J Nutr 2009;48:315-322. doi: 10.1007/s00394-0090017-y. 